\section{Andamios Revista de Investipación Social}

\section{Andamios. Revista de Investigación Social} ISSN: 1870-0063

revistaandamios@uacm.edu.mx

Universidad Autónoma de la Ciudad de México México

Pereyra, Guillermo

Heterogeneidad, improductividad y ocio

Andamios. Revista de Investigación Social, vol. 3, núm. 6, junio, 2007, pp. 217-237

Universidad Autónoma de la Ciudad de México

Distrito Federal, México

Disponible en: http://www.redalyc.org/articulo.oa?id=62830609

- Cómo citar el artículo

Número completo

- Más información del artículo

Página de la revista en redalyc.org

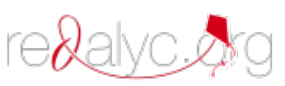

Sistema de Información Científica

Red de Revistas Científicas de América Latina, el Caribe, España y Portugal

Proyecto académico sin fines de lucro, desarrollado bajo la iniciativa de acceso abierto 


\title{
HETEROGENEIDAD, IMPRODUCTIVIDAD Y OCIO
}

\author{
Guillermo Pereyra*
}

\begin{abstract}
RESUMEN. La globalización articula una enorme complejidad socioeconómica con formas de organización de la cooperación que incluyen amplias masas de desocupados, pobres y "nuevos pobres". En otras palabras, estamos frente a una heterogeneidad socioeconómica que no puede ser fácilmente procesada por las instituciones vigentes. En este contexto surge una paradoja: aunque las nociones de productividad y de cooperación social siguen siendo fundamentales en la organización de las instituciones socioeconómicas contemporáneas, su dislocación se hace cada vez más visible como consecuencia del aumento del desempleo y del tiempo de ocio, esto es, de la improductividad. Este trabajo tiene como finalidad abordar esta paradoja, y para ello se analizan las relaciones que la teoría marxista y liberal establecen entre la cooperación social, la productividad y el ocio.

Palabras clave. Cooperación social, justicia distributiva, heterogeneidad, improductividad, ocio.
\end{abstract}

VIVIMOS EN SOCIEDADES EXTREMADAMENTE complejas, donde las instituciones socioeconómicas cambian a ritmos vertiginosos. Esta complejidad convive con una organización de la cooperación social que incluye amplias masas de desocupados, marginales, pobres y "nuevos pobres". En otras palabras, en un mundo donde las dislocaciones de los mercados son más recurrentes y severas en sus efectos, cada vez son más las personas que pasan a formar parte de lo que en alguna época se llamó el "lumpenproletariado". Debido a esto, estamos siendo testigos de una heterogeneidad socioeconómica que no puede ser fácilmente procesada por

\footnotetext{
* Maestro en ciencias sociales (Facultad Latinoamericana de Ciencias Sociales, Sede Académica de México, 2006). Estudiante de doctorado en ciencias sociales de la Flacso. Correo electrónico: guillermodpereyra@yahoo.com.mx
}

Volumen 3, número 6, junio, 2007, pp. 217-237 
Guillermo Pereyra

las instituciones vigentes de la cooperación social. La marginalidad no sólo tiene como referente el desempleo fluctuante del sistema fabril, sino también una variedad de situaciones que cubren el movimiento global de la población dentro de mercados fragmentados y débilmente protegidos. Parafraseando a Georges Bataille (2000: 127), en un mundo globalizado y crecientemente homogeneizado por el capital, lo heterogéneo es todo lo que resulta del "gasto improductivo".

En el presente trabajo pretendo indagar, desde una perspectiva de análisis posestructuralista, la dislocación de sentido que ejerce el aumento de la improductividad y del ocio que caracteriza al capitalismo globalizado sobre la idea general de que los arreglos sociales cooperativos deben ser productivos. Para abordar la manera dilemática en que conviven estas dos cuestiones, el argumento se estructura en tres partes. En el primer apartado presento una breve síntesis del modo como el posestructuralismo plantea la cuestión de "lo heterogéneo". A partir de estos supuestos, estaré en condiciones de discutir la heterogeneidad dislocatoria que representa la improductividad y el ocio generalizado con respecto a toda estructura cerrada u ordenada de cooperación social. En la segunda sección indago el lugar central que tiene la idea de cooperación social productiva en la justicia distributiva tanto liberal como marxista. El objetivo que allí me propongo es mostrar la analogía que existe entre el marxismo y el liberalismo en la forma en que los improductivos son expulsados de sus respectivos esquemas de justicia distributiva. De la igual valoración que ambas teorías hacen de la heterogeneidad improductiva se desprende un conjunto de afirmaciones que me permitirán analizar en la tercera parte la situación de ocio generalizado. La principal conclusión será que el ocio generalizado no constituye un momento excepcional o marginal de la estructura de la cooperación social, sino más bien un elemento fundamental en su configuración.

EL POSESTRUCTURALISMO Y LA HETEROGENEIDAD

Comenzaré este trabajo con una discusión teórica que permita abordar el fenómeno de la improductividad entendido como una expresión de lo heterogéneo. En términos generales, la heterogeneidad constituye una 
amenaza para la unidad del pensamiento racional y, por ello, sólo comienza a ser teorizada sistemáticamente con la puesta en duda de toda idea de necesidad incondicionada. En este contexto, desde diferentes perspectivas un conjunto de autores contemporáneos se ha dedicado a la tarea de abordar este fenómeno. En lo que sigue, me concentraré en sólo dos propuestas provenientes del posestructuralismo, a saber: la teoría de la différance de Jacques Derrida y la noción de heterogeneidad social desarrollada en la teoría de la hegemonía de Ernesto Laclau.

Grosso modo el posestructuralismo parte del supuesto de que los sistemas de sentido dependen de exteriores discursivos que parcialmente constituyen dichos órdenes, mientras que potencialmente los subvierten. Esto quiere decir que algo constitutivamente heterogéneo al sistema o estructura social tiene que estar presente en esta última desde el mismo comienzo, impidiéndole constituirse como totalidad cerrada o representable (Laclau, 2002). El avance que supone el posestructuralismo con respecto a los programas estructuralistas radica en que los últimos no podían dar cuenta convenientemente de la contingencia de los vínculos sociales. Es decir, aunque los estructuralistas habían señalado que todo sistema no es más que un conjunto relacional de posiciones diferenciales, seguían postulando la posibilidad de reconstruir un sistema cerrado, esto es, capaz de controlar todo exceso heterogéneo a su propia lógica interna (Laclau, 1988). En este marco, la propuesta de Derrida se fundamenta en una ontología de lo heterogéneo que está en la base de la constitución de toda estructura. Según esto, la différance es la heterogeneidad que hace posible y, a la vez, imposible determinar los límites de una totalidad de sentido (Derrida, 2003b: 39-62). Para dar cuenta de este juego paradójico de posibilidades e imposibilidades, es preciso tener en cuenta dos tesis básicas de la différance. Por un lado, ésta "no es ni una palabra ni un concepto" porque es "la posibilidad de la conceptualidad, del proceso y del sistema conceptuales en general" (Derrida, 2003b: 46); en otras palabras, es su condición de posibilidad porque es la exterioridad discursiva que permite "fijar" los límites internos del sistema conceptual. Pero, por otro lado, es aquello que hace que dicho sistema sea una totalidad opaca de significación porque subvierte permanentemente el valor de presencia del signo, introduciendo un espaciamiento y una temporización constitutiva. Este es el sentido que 
Guillermo Pereyra

Derrida explora en la palabra "diferir", en tanto sinónimo de "ser diferente" y de "retraso, demora, rodeo". La deconstrucción opera difiriendo las dicotomías diferenciales de la metafísica occidental mostrando que, lejos de haber una relación de subordinación jerárquica o de complementación entre ambos términos, lo que existe entre ellos es la imposibilidad última de un espacio común (lógica del espaciamiento) que los reúna en una armonía de significación (lógica de la temporización) (Derrida, 2003b: 43-44). En resumen, el signo es siempre una presencia "diferida", en fuga constante a lo largo de un espacio no lineal, sin principio ni fin.

La noción de heterogeneidad social que Laclau desarrolla en sus últimos trabajos es una forma de traducir al lenguaje de la teoría social la lógica de la différance derrideana. El punto de partida de la teoría de la hegemonía de Laclau es la construcción de unidades o cadenas equivalenciales de sentido que resultan de la articulación de una serie de demandas particulares insatisfechas. Esta articulación política es lo que permite dar forma a los campos discursivo-antagónicos presentes en la sociedad. Pero esto no quiere decir, según Laclau, que toda demanda pueda ser representada por tales equivalencias:

una demanda puede no ser incorporada a la cadena equivalencial porque se opone a los objetivos particulares de demandas que ya son eslabones de esa cadena. Si el particularismo de las demandas individuales fuera totalmente neutralizado por su inscripción equivalencial, esta posibilidad podría ser descartada, pero sabemos que esto no ocurre. Por lo tanto, una cadena equivalencial no sólo se opone a una fuerza o un poder antagónico, sino también a algo que no tiene acceso a un espacio general de representación. (Laclau, 2005: 175)

La demanda inarticulada, que es el contenido de una heterogeneidad social incontrolada, es entonces aquella que no está incluida en un espacio común de diferencias articuladas equivalencialmente. En palabras de Laclau: "La heterogeneidad [social] [...] no significa diferencia; dos entidades, para ser diferentes, necesitan un espacio dentro del cual esa 
diferencia sea representable, mientras que lo que ahora estamos denominando heterogéneo presupone la ausencia de ese espacio común" (Laclau, 2005: 176). La heterogeneidad es aquello que hace que el campo de la representación "sea un espejo turbio y roto, interrumpido constantemente por un "real" heterogéneo al cual no puede dominar simbólicamente". Como consecuencia de esta presencia dislocatoria de lo heterogéneo, la teoría de la hegemonía "renuncia a la idea de un espacio saturado y de una representabilidad plena" (Laclau, 2005: 177).

¿Qué consecuencias se derivan de las afirmaciones anteriores? Si la heterogeneidad es, por definición, aquello que no puede ser incorporado en un espacio simbólico cerrado, se trata entonces de una demanda definida por su singularidad, ${ }^{1}$ por aquello "tan particular" que no tiene medida común con nada similar. Esto exige desligar lo heterogéneo de la idea de una positividad monádica cerrada en sí misma; lo heterogéneo no es aquello que permanece totalmente aislado en un espacio de representación, sino más bien es lo que pugna por su inclusión imposible. De esta manera, constituye una singularidad improcesable, cuyo exceso subvierte las posibilidades de fijar racionalmente los límites simbólicos de una estructura.

Habiendo expuesto los elementos básicos que definen lo heterogéneo, estamos en condiciones de abordar el papel que juega la heterogeneidad improductiva en dos propuestas diferentes de la justicia, a saber: la marxista y la liberal.

MARX y RAWLS: LA EXCLUSIÓN DE LOS IMPRODUCTIVOS

El marxismo constituye, además de un modo de entender la realidad y la historia, una propuesta normativa. Ésta consiste en afirmar que es posible determinar en el capitalismo una estructura de explotación que

\footnotetext{
${ }^{1}$ Lo singular es una noción recurrente en los estudios posestructuralistas y, grosso modo, es homologable a la idea lacaniana de "acto ético". En palabras de Joan Copjec, lo singular está asociado a "la denigración de toda noción de una instancia anterior o superior que pudiera prescribir o garantizar el acto. Alma, eternidad, absoluto o poder patriarcal son nociones que deben ser destruidas para que un acto pueda considerarse único y capaz de autoimponerse su propia necesidad" (Copjec, 2006: 43-44).
} 
Guillermo Pereyra

resulta éticamente inaceptable, y que será superada en el momento de cancelación del conflicto de clases que representa el comunismo. Como es sabido, el comunismo es aquella instancia que será regida por el principio de distribución "De cada cual según sus capacidades, a cada cual según sus necesidades". Sin embargo, ese principio no parece tratar a todos con igual consideración. Prestemos atención a la siguiente afirmación de Phillipe Van Parijs:

En la sociedad socialista (ideal) [...] los medios de producción son detentados colectivamente por los trabajadores, que deciden qué parte del producto social se dedica a la acumulación y la manera en que el saldo será distribuido entre los ciudadanos. Por supuesto, algunos no trabajadores pueden recibir una parte del producto —los jóvenes, los viejos, los enfermos, aun las amas de casa y (si queda algo) los mendigos-, pero únicamente en virtud de la benevolencia de los trabajadores y no porque tengan algo que ofrecer a cambio. (Parijs, 1993: 83)

Es decir, en el marxismo hay un grupo de personas que la justicia excluye por definición: aquellos que no cooperan con su trabajo, sea por el motivo que fuera, en la generación del producto social. Pero para que esa exclusión opere en el comunismo, éstos deben estar desde el inicio excluidos de los criterios normativos marxianos; incluso antes de la llegada del comunismo, quienes no trabajan deben quedar fuera del alcance lo que se considera "lo correcto", "lo permitido" o "lo razonable".

Esta última cuestión se observa muy claramente en El dieciocho Brumario de Luis Bonaparte. Allí Marx define con desprecio al lumpenproletariado, y la lectura deconstructiva realizada por Peter Stallybrass (citado en Laclau, 2005: 180-184) muestra que Marx nunca puede delimitar conceptualmente a esta clase social. En otras palabras, Marx desprecia algo que no puede definir, algo que no parece tener límites conceptuales claramente definidos. En el siguiente pasaje, el filósofo alemán intenta caracterizar a quienes eran favorecidos por la sociedad de beneficencia creada por Bonaparte y que llevaba por nombre Sociedad del 10 de Diciembre: 
Junto a roués [libertinos] arruinados, con equívocos medios de vida y de equívoca procedencia, junto a vástagos degenerados y aventureros de la burguesía, vagabundos, licenciados de tropa, saltimbanquis, lazzaroni, carteristas y rateros, jugadores, alcahuetes, dueños de burdeles, mozos de cuerda, escritorzuelos, organilleros, traperos, afiladores, caldereros, mendigos; en una palabra, toda esa masa informe, difusa y errante que los franceses llaman la bohéme; con esos elementos, tan afines a él, formó Bonaparte la solera de la Sociedad del 10 de Diciembre, "Sociedad de beneficencia" en cuanto que todos sus componentes sentían, al igual que Bonaparte, la necesidad de beneficiarse a costa de la nación trabajadora. (Marx, 1978: 137)

Los insultos no se detienen: Marx afirma a renglón seguido que Bonaparte se erige en representante de "esta hez, desecho y escoria de todas las clases" (1978: 137). El lumpenproletariado constituye, de esta forma, una masa de improductivos que no hacen más que recibir los beneficios de quienes cooperan y trabajan decentemente. Muchos años después encontramos una opinión similar sobre los improductivos en boca de David Schmidtz (2000: 96), un liberal anarco-capitalista: "El sistema que minimiza la indigencia es un sistema según el cual los ingresos de una persona dependen de que se produzca algo que los demás valoran. Esto es así, por mucha compasión que sintamos por las personas con graves minusvalías. Es así, digan lo que digan las teorías sobre dependencia o explotación o sobre los defectos de los mercados laborales".

¿Qué hay de equivalente entre las afirmaciones de Marx y las del liberal conservador de nuestra época? ¿Qué es lo que tanto fastidia a teóricos tan disímiles como Marx y Schmidtz? Lo que a primera vista molesta a ambos no es que el improductivo no produzca nada en la sociedad, sino que más bien se trata de alguien que no es apto para en-

${ }^{2}$ El trabajo de Schmidtz abunda en opiniones en contra de garantizar subsidios a hombres y mujeres estadounidenses que según él cayeron en desgracia irresponsablemente (mujeres embarazadas, desocupados, etcétera) y que los ciudadanos-contribuyentes no tienen por qué mantener por medio de sus impuestos. 
trar en lógicas equivalenciales de intercambio porque lo que producen no es valorado por otros. No es, por lo tanto, que éste sea visto con desprecio porque no genera nada; de hecho es considerado así porque "produce demasiado", es decir, algo que sobrepasa los límites razonables de lo socialmente útil. El improductivo es capaz de generar con su "producto no productivo" una cesura radical en la lógica del intercambio y de la administración de las cosas "naturalmente" consideradas. En pocas palabras, introduce en la estructura normal de la producción y distribución un desorden que no puede ser controlado. Esto hace que los improductivos tengan un estatuto ontológico paradójico: por un lado, representan una falta en el interior de la estructura porque se sustraen a toda cuenta distributiva; son los que deben ser excluidos para que el reparto del producto social tenga lugar entre personas trabajadoras y cooperativas. En tanto "hez, desecho y escoria" son expulsados hacia los bordes de la sociedad. Pero, por otro lado, los improductivos representan un exceso dentro de la organización de la distribución, algo que es tan amplio y amorfo que no puede ser aprehendido con total precisión. Lo que particularmente incomoda a Marx de Bonaparte es que alienta la institución de un modo de relaciones sociales enteramente singular, y que esto implique a su vez un conjunto de desplazamientos heterogéneos radicales que el bonapartismo traía consigo: de la "tragedia" a la "farsa" en el dominio de las clases propietarias (Marx, 1978: 95); de la dominación del "partido del orden" burgués a la institucionalización de la "comedia"; de la digna lucha de clases entre la burguesía y el proletariado a la centralidad política y estructural de esa multitud de haraganes que por justicia no tienen por qué ser beneficiados. En palabras de Marx: "[Bonaparte] concibe la vida histórica de los pueblos y los grandes actos de Gobierno y de Estado como una comedia, en el sentido más vulgar de la palabra, como una mascarada, en que los grandes disfraces y las frases y gestos no son más que la careta para ocultar lo más mezquino y miserable" (Marx, 1978: 137). "Farsa", "comedia", "disfraces" y "gestos": en ello se condensa la irrupción del exceso singular del lumpenproletariado en el interior de la estructura distributiva que la "seriedad marxiana" no puede comprender.

No me interesa cuestionar éticamente si Bonaparte era o no de hecho un farsante o un estafador de las masas. Lo que quiero destacar es el 
carácter paradójico de la justicia marxiana, pues aunque se plantea como una virtud universal - para el proletariado en tanto que encarna sin mediaciones los intereses de la humanidad- debe excluir a los improductivos como su propia condición de posibilidad. Lo mismo ocurre, como puede imaginarse, en el nivel de la dialéctica de la historia, y esto ha sido subrayado por Laclau (2005: 181): "el carácter de "extranjero" puro del lumpenproletariado, su expulsión del campo de la historicidad, es la condición misma de posibilidad de una interioridad pura, de una historia poseedora de una estructura coherente [como proceso productivo]".

Consideremos ahora el planteo liberal de la teoría de la "justicia como equidad" de John Rawls. Comencemos exponiendo la primera analogía que existe entre ésta y el marxismo. Aunque durante mucho tiempo el marxismo y el liberalismo constituyeron discursos mutuamente incontaminados, Rawls no considera esta incompatibilidad como algo esencial. Según él, "una sociedad bien ordenada en la que se satisface la condición de plena publicidad [...] será una sociedad sin ideología (entendida en el sentido de la falsa conciencia de Marx)" (Rawls, 2002: 166-167). Como en la sociedad gobernada por los principios de la justicia operan los criterios kantianos de la publicidad de los actos públicos, nada puede permanecer enmascarado por el poder de quienes operan en las sombras. La sociedad rawlsiana es una sociedad que puede llegar a niveles deseables y posibles de transparencia política. Pero al igual que en el caso de Marx, la transparencia de la estructura de la justicia se logra gracias a la exclusión de una singularidad heterogénea. Para respaldar esta tesis es preciso responder dos preguntas: 1) ¿qué es lo que excluye específicamente la justicia como equidad?; 2) si la justicia es una virtud universal, ipuede incluirse posteriormente lo excluido en una futura estructura de distribución?

Antes de responder estas preguntas, es necesario que nos detengamos en un supuesto básico de la justicia como equidad. Según Rawls, la idea de cooperación social, en tanto artificio normativo fundamental, presupone la noción de productividad — si no existiese cooperación productiva, no habría nada que distribuir (Barry, 2001: 258)—. Que los arreglos sociales sean productivos significa que "no son, por así decirlo, un juego de suma cero en el que la ganancia de una persona (o de un 
grupo) es la pérdida de otra" (Rawls, 1999: 173). La cooperación productiva implica, naturalmente, que los ciudadanos de una sociedad bien ordenada son miembros cooperativos capaces de producir cosas. Esto implica que:

todos tienen facultades intelectuales suficientes para desempeñar un papel normal en la sociedad, y que ninguno adolece de necesidades inusuales especialmente difíciles de satisfacer, por ejemplo, exigencias médicas inusuales y costosas. Por supuesto, cuidar de los que tienen tales exigencias es una cuestión prácticamente acuciante. Pero en este estadio inicial el problema fundamental de la justicia social surge entre personas que participan en forma plena, activa y moralmente consciente en la sociedad, y que están directa o indirectamente asociadas entre sí a lo largo de toda una vida completa. (Rawls, 1999: 238)

De este modo, Rawls considera que en el "estado inicial" donde la sociedad comienza a dar sus primeros pasos en el camino de la institucionalización de la justicia resulta "sensato dejar a un lado ciertas complicaciones difíciles" como la enfermedad u otra limitación que imposibilite la cooperación de algunos (Rawls, 1999: 238). Como puede verse, estamos ya en condiciones de responder a nuestra primera pregunta: para que la sociedad justa sea un sistema cerrado, y evitar así un continuo desplazamiento de significados sobre lo que significa ser un sujeto político de la justicia, es necesario excluir desde el comienzo la idea de improductividad. En este punto, si seguimos los planteos de C. B. Macpherson (1991: 24) y de Gerald Cohen (2001: 182), lo que parece estar sosteniendo Rawls es que el requisito básico para que una persona sea un sujeto de derecho no se debe tanto a su condición de ciudadano libre e igual, sino a su potencial capacidad de miembro productivo de una sociedad de mercado. Ya lo decía Marx: una "nación trabajadora" merece desde un principio un trato diferente (y deferente) respecto del grupo incontable e inútil de improductivos. Pero Rawls trata de resolver este problema afirmando que es posible destinar recursos sociales a la salud normal y a las necesidades médicas de los no 
productivos "en el estadio legislativo a la luz de las condiciones sociales existentes y de las expectativas razonables relativas a la frecuencia de enfermedades o accidentes" (Rawls, 1999: 272). ${ }^{3}$ Sin embargo, ipor qué una teoría de la justicia que considera prima facie como sujetos de la justicia a los miembros productivos tendría la obligación de extenderse luego a los no productivos? ¿Sobre la base de qué justificación? ¿Por qué incluir luego algo que en un principio se excluye? El mismo Rawls no oculta sus dudas al respecto:

Considero evidente, y aceptado por el sentido común, que tenemos un deber para con todos los seres humanos, por muy gravemente discapacitados que estén. La cuestión tiene que ver con el peso que damos a esos deberes cuando entran en conflicto con otras exigencias básicas. En algún punto, pues, debemos ver si la justicia como equidad puede extenderse para que proporcione directrices para esos casos; y, de lo contrario, si debe ser rechazada en vez de complementada por alguna otra concepción [...]. Yo no sé hasta dónde puede extenderse con éxito la justicia como equidad para que cubra los tipos más extremos de casos.

(Rawls, 2002: 232-233)

El problema de fondo es, en definitiva, un problema de índole retórico acerca de si Rawls admite o no un desplazamiento metonímico — lo que él llama la "extensión" de la justicia como equidad-que amplíe el juego de la significación sobre lo que define a un agente legítimo de la justicia. Este Rawls - el que duda sobre el alcance de su teoría e implícitamente invoca una solución retórica que no quiere encarar-, no tiene mucho que ver con el Rawls categórico y (cuasi)kantiano de la

\footnotetext{
${ }^{3}$ Para una presentación de las etapas en las que se van adoptando los principios de la justicia — posición original, convención constituyente, etapa legislativa y etapa de la "administración de las cosas"-, véase Rawls (2002: 79-80).

${ }^{4}$ Para un estudio detallado de los inconvenientes que le genera a la teoría de la justicia como equidad este tipo de extensiones ad hoc para los casos de la justicia internacional e intergeneracional, véase Barry (2001, 197-230).
} 
posición original y del que confecciona la lista acotada de los bienes primarios. Esta indeterminación no es compatible con la intención de una teoría que tiene por objeto "aclarar" y "organizar" nuestros juicios acerca de la justicia, o reducir al máximo posible los desacuerdos razonables (Rawls, 1996a: 31). En definitiva, lo que hace Rawls es desplazar (o aplazar) el desplazamiento de significados; lo es equivalente a subsumir su teoría de la justicia a la lógica de la différance, al mismo tiempo que niega el papel de las demandas heterogéneas de los improductivos. Tengamos presente los sentidos que asume la noción de différance, y que ya fueron presentados. En primer lugar, la justicia del estadio inicial difiere (o es distinta) de la justicia del estadio legislativo: la segunda es más incluyente que la primera. En segundo lugar, diferir también significa "esparcir" o "diseminar", y esto es lo que precisamente la justicia como equidad quiere evitar cuando expresa sus dudas sobre la extensión futura de la justicia inicial. Lo que elude, pues, es una diseminación semántica sobre sus límites y su alcance; una postergación de la consideración de la singularidad individual o de los casos particulares de improductividad. Y por último, diferir es sinónimo de "retraso", "demora", "posposición", y es lo que visiblemente Rawls propone: postergar para un futuro incierto la extensión igualitarista de su concepción de la justicia. Pero ipor qué ser ampliamente justos mañana cuando podríamos serlo hoy?

Esta indeterminación termina minando las bases conceptuales del argumento rawlsiano, porque los improductivos pasan a ser objeto de un deber humanitario similar a una idea de caridad que se opone claramente a la obligación moral fundada en la justicia política. En Teoría de la justicia, Rawls (1997: 115-118) distinguía entre los deberes naturales aplicables a las personas - por ejemplo: la ayuda mutua, evitar la crueldad, etcétera- y los deberes de la justicia institucional que se predican de la estructura básica de la sociedad, pero mantener esta distinción luego del giro "no metafísico" que dio su teoría en Liberalismo político es problemático por dos motivos. Primero, porque sostener la existencia de deberes naturales lo compromete con una noción de naturaleza humana que él mismo rechaza. Segundo, porque ese deber natural tendría que ser considerado en el estadio legislativo, esto es, en un contexto donde se fomenta la neutralidad con respecto a cualquier 
tipo de doctrina comprehensiva o metafísica. En otras palabras, la justicia política que Rawls defiende terminaría siendo análoga a aquello que ella misma desdeña, a saber: una justicia basada en deberes naturales intrínsecos a las personas. Entre la benevolencia y la justicia, entre la compasión y el derecho: al igual que en el esquema marxista, los improductivos rawlsianos ocupan un lugar difuso que termina siendo un no-lugar; una interioridad ("en el estadio legislativo se atenderán sus expectativas") que tiene un momento primario de radical exterioridad ("en el estadio inicial quedan fuera de la justicia").

Hasta ahora hemos puesto la atención en la dimensión de futuro para caracterizar esta exclusión originaria. ¿Qué se puede decir del pasado de los improductivos en el momento en que son "interceptados" por la justicia? Una cuestión clave para responder esto es que Rawls considera que en las sociedades modernas de mercado existen inevitablemente distintas posiciones sociales y económicas, esto es, diferentes estándares de vida y de riqueza. Esas diferencias están "determinadas, en parte, tanto por el sistema político como por las circunstancias económicas y sociales"; y tales instituciones habrán de favorecer "ciertas posiciones iniciales frente a otras" (Rawls, 1997: 20). En este contexto, el principio de la diferencia ${ }^{5}$ representa un acuerdo original que tiene por objeto "mitigar en lo posible los hándicaps arbitrarios que resultan de nuestras posiciones iniciales de partida en la sociedad" (Rawls, 1999: 132). De este modo, la "posición inicial" en la que se encuentran los miembros de una determinada sociedad es el punto de referencia primario que es necesario considerar para lograr una distribución equitativa del producto social. Rawls expresa con más precisión esta idea en el siguiente pasaje: "[las] ventajas contingentes e influencias accidentales que derivan del pasado no debieran afectar ningún acuerdo basado en los

\footnotetext{
${ }^{5}$ El principio de la diferencia es un principio igualitarista que establece lo siguiente: "Las desigualdades sociales y económicas sólo se justifican por dos condiciones: en primer lugar, estarán relacionadas con puestos y cargos abiertos a todos, en condiciones de justa igualdad de oportunidades; en segundo lugar, estas posiciones y estos cargos deberán ejercerse en el máximo beneficio de los integrantes de la sociedad menos privilegiados" (Rawls, 1996b: 31).
} 
Guillermo Pereyra

principios reguladores de las instituciones de la estructura básica misma, partiendo del presente, hacia el futuro" (Rawls, 2002: 40).

En esta última referencia es posible advertir que ahora Rawls encara el presente y el futuro con mucho menos vacilaciones que hasta hace un momento con los improductivos. No obstante, lo que ahora queda negado —e igualmente diferido o aplazado en la reflexión- es la dimensión del pasado. De esto da cuenta Alasdair MacIntyre cuando afirma que en la justicia como equidad es "irrelevante cómo llegaron a su situación actual los que ahora se hallan en grave necesidad; la justicia es asunto de modelos presentes de distribución, para los que el pasado es irrelevante" (MacIntyre, 2001: 305). Esta negación del pasado de los sujetos que se hallan en "grave necesidad", ¿no es otra forma de expresar el mismo desinterés que el propio Marx tiene sobre la "equívoca procedencia" del lumpenproletariado?: "[El lumpenproletariado es] un tropel de bribones, del mejor de los cuales puede decirse que no se sabe de dónde viene" (Marx, 1978: 179). Parafraseando a Derrida, si tanto el lumpenproletariado como los improductivos deben permanecer en el anonimato es para no advertir la "singularidad de la exposición noeconómica del otro" (Derrida, 2003a: 36).

El EMPLEO DEL TIEMPO: EL CARÁCTER SINGULAR DEL OCIO

En el filme El empleo del tiempo (L'Emploi du temps, 2001), de Laurent Cantent, Vincent es despedido de su trabajo de consultor de una empresa y finge durante varios meses tener un nuevo empleo como funcionario de Naciones Unidas. Su despido fue ocasionado por las constantes faltas a su trabajo o, como él mismo se lo confiesa a un amigo, porque lo único que lo entusiasmaba del mismo eran los viajes que hacía en su coche. Para sobrevivir o "ganar tiempo", les pide dinero a sus padres, vende artículos clandestinos y le miente a sus amigos haciéndoles creer que coloca su dinero en mercados financieros emergentes de países excomunistas. Lo que busca Vincent es tiempo libre, o no tener que estar atado a las obligaciones del trabajo, pero al final de la película termina teniendo una entrevista de trabajo. Lo que él no ha podido trascender, en definitiva, es la estructura de la cooperación social, y su fracaso 
o derrota final se debe en parte a que nadie puede comprender por qué el trabajo lo cansa o lo agobia. Se trata de una experiencia tan privada y singular que ni siquiera puede ser comprendida por sus vínculos familiares más cercanos. Si Vincent ha vivido deshonrosamente del dinero y del esfuerzo de los demás, la entrevista final de trabajo muestra también que el Gran Otro -la estructura de la cooperación socioeconómica - tampoco puede vivir sin su trabajo. En suma, El empleo del tiempo aborda la singularidad inarticulable de la experiencia del ocio y del cansancio que produce el trabajo que, por otro lado, resulta tener un poder dislocatorio cuando se extiende más allá de los rigurosos tiempos de la cooperación social.

En Las esferas de la justicia, Michael Walzer analiza detenidamente el carácter singular que define al tiempo libre. El capítulo dedicado a ello comienza con una síntesis de los diversos significados que ha tenido el ocio, destacando particularmente dos. El primero está asociado a la filosofía aristotélica y, de acuerdo con ella, el ocio consiste en "no tener objetivos" o no estar atado a metas fijas; el segundo es el sentido marxista condensado en la idea del autocontrol o "control libre" del trabajo. Pero en las sociedades complejas contemporáneas, el ocio se vuelve algo más diverso en su significación de lo que pensaron los autores clásicos:

El tiempo libre no tiene una única estructura moral o justamente necesaria. Lo moralmente necesario es que su estructura, sea cual fuere, no sea deformada por lo que Marx llamó las "usurpaciones" del capital, o por el fracaso de la previsión comunitaria cuando ésta haya sido necesaria, o por la exclusión de los esclavos, extranjeros y parias. Libre de estas deformaciones, el tiempo libre será experimentado y disfrutado por los miembros de una sociedad libre en todas las distintas formas que ellos pueden inventar colectiva o individualmente. (Walzer, 1997: 207)

En este contexto, la teoría de la justicia de Rawls es consciente de la expresión heterogénea que puede adquirir el ocio en las sociedades actuales y, por ello, busca limitar racionalmente sus efectos dislocatorios. Como ya vimos, Rawls parte de la idea de que "todos los ciudadanos 
son miembros normales y plenamente cooperativos de la sociedad durante una vida completa" (Rawls, 2002: 236). Pero esta afirmación genera dudas sobre la importancia o no del tiempo de ocio: si éste es considerado razonablemente como un derecho humano que es legítimo proteger, todo el mundo debería tener acceso a un poco de tiempo libre en sus vidas. El problema es qué cantidad de ocio puede admitir la estructura cooperativa, esto es, dónde habrá de fijarse un límite razonable más allá del cual se vuelve un exceso perjudicial para la sociedad. Para abordar esto, Rawls se detiene en la relación que existe entre el principio de la diferencia, los menos aventajados y su tiempo libre. Si los menos aventajados son aquellos que reciben una serie de prestaciones sociales con motivo de su condición desfavorable, iesto no puede incentivar una conducta excesivamente ociosa por parte de ellos? Rawls mismo se hace esta pregunta: " $i S o n$ los menos aventajados [...] los que viven de las prestaciones de bienestar y se pasan el día haciendo surf en Malibú?" (2002: 236). El filósofo norteamericano encuentra dos maneras de tratar esta cuestión. La primera consiste en asumir que "todo el mundo trabaja una jornada laboral convencional". La segunda supone incluir "en el índice de bienes primarios una determinada cantidad de tiempo de ocio, digamos que dieciséis horas al día si la jornada laboral convencional es de ocho horas". Por consiguiente, los que no trabajan "tienen ocho horas adicionales de ocio y consideramos esas ocho horas adicionales como equivalentes al índice de los menos aventajados que sí trabajan una jornada convencional. Los surfistas deben de algún modo mantenerse a sí mismos" (Rawls, 2002: 236-237).

En lo que sigue, extraeremos las consecuencias que se siguen del planteo rawlsiano. Las reuniremos en cuatro momentos argumentativos.

1. Rawls, a diferencia de Walzer, propone una base objetiva para medir el ocio: dieciséis horas diarias. ${ }^{6}$ Como puede observarse, el exceso

${ }^{6}$ Walzer se opone, por ejemplo, al Acuerdo Internacional para los Derechos Económicos, Sociales y Culturales de Naciones Unidas, que incluye en su lista de derechos días festivos y vacaciones. Según él, "esto no es definir derechos humanos, sino simplemente proponer un conjunto particular de medidas sociales, que no es el mejor por fuerza, o lo mejor, para cada sociedad y cultura. El derecho que requiere protección es, por completo, de otra especie: no ser excluido de las modalidades de descanso central al 
puede ser determinado con precisión: los que no trabajan deben autosostenerse, y es injusto que la sociedad tenga que cargar con su irresponsabilidad. ${ }^{7}$

2. Dada la prioridad innegociable del primer principio de la justicia, ${ }^{8}$ una sociedad justa que protege la libertad debería ser compatible con la existencia de individuos más y menos aventajados que asuman un plan de vida completamente dedicado al ocio (a nadie se le puede obligar por la fuerza a abandonar ese tipo de vida). Pero aunque son libres de hacerlo, deben mantenerse a sí mismos, y en el caso particular de los menos aventajados no tienen derecho a recibir ninguna compensación estatal.

3. Como ya hemos visto, si la justicia favorece un amplio esquema de libertades individuales con el propósito de que todo el mundo consiga fomentar su propia concepción del bien, entonces se reconoce la libertad de poder llevar una vida de tiempo libre total. Sin embargo, si esta situación se extiende a todos o a una muy amplia cantidad de individuos, la sociedad bien ordenada se colapsa: la justicia perdería sentido no sólo porque no habría ningún producto que repartir, sino porque además no habría vínculos sociales estables que permitan el compromiso con la justicia. Se llegaría a aquella situación en la cual las libertades de unos entran con colisión con las de otros, o en la que el perjuicio es superior a lo que se gana individualmente. Pero como el sujeto rawlsiano es razonable, es lógico que tienda a rechazar esta situación de anomia social. En conclusión, el ocio total sólo puede ser aceptado en casos aislados o puede ser disfrutado por unos cuantos a quienes, además, se les exigirá que no hagan un uso indebido del producto de la cooperación.

propio tiempo y lugar de uno, disfrutar de vacaciones (aunque no de las mismas vacaciones) si éstas poseen importancia especial, participar en los festivales que dan forma a la vida común dondequiera que exista una vida común" (Walzer, 1997: 207).

${ }^{7}$ Para una afirmación similar a ésta, véase Dworkin (2003: 12).

${ }^{8}$ El primer principio está asociado a la idea de libertad y establece lo siguiente: "Cada persona tiene igual derecho a exigir un esquema de derechos y libertades básicos e igualitarios completamente apropiado, esquema que sea compatible con el mismo esquema para todos; y en este esquema, las libertades políticas iguales y sólo esas libertades, tienen que ser garantizadas en su valor justo" (Rawls, 1996b: 31). 
Guillermo Pereyra

El resultado que se sigue de esto es que Rawls hace equivalentes dos situaciones que son inequivalentes, o que son heterogéneas entre sí: mientras el más aventajado totalmente ocioso puede no trabajar y, aún así, vivir muy cómodamente sin necesidad de la ayuda estatal, el menos aventajado igualmente ocioso está obligado a hacerlo. Ahora bien, Rawls sostiene que el supuesto del carácter plenamente cooperativo de los ciudadanos implica "que todos están dispuestos a trabajar y hacer la parte que les toca en punto a compartir las cargas de la vida social" (Rawls, 2002: 236). Hace falta nada más que un poco de conciencia socialista —no necesariamente marxista, en los términos antes definidos - ${ }^{9}$ para darse cuenta de que, a pesar de que todos están igualmente obligados a trabajar, el menos aventajado está más constreñido por su situación desventajosa a trabajar que el más aventajado. Mejor dicho, aunque los dos deben cooperar para poner en marcha la sociedad bien ordenada, la diferencia radica en que el más aventajado puede exceder con mayor libertad el límite del tiempo de ocio que Rawls considera razonable.

4. Lo anterior nos lleva directamente a la última de las consecuencias que rigen la situación del ocio generalizado. Expliquémosla por medio de tres afirmaciones: 1) si el ocioso es, por definición, alguien que depende de otros para vivir y 2) si la sociedad mercantil es aquella en la cual todos dependen de todos para conseguir los productos de la cooperación, entonces 3) el ocio total es el "reverso ominoso" de la situación cooperativa, puesto que el mercado —o el capital— depende del esfuerzo de todos para lograr el éxito de sus operaciones. Esto está presente claramente, como decíamos, al final de El empleo del tiempo: si Vincent ha sido un ocioso que se aprovechó de sus padres y amigos, el mercado es aún más ocioso que él porque necesita de ese engranaje particular — y de muchos otros más- para consumar sus propósitos de dominación. Por consiguiente, el ocio total no debería ser entendido tanto como la situación donde nadie coopera con los demás o no se produce absolutamente nada, sino más bien

${ }^{9}$ Sobre las diferencias entre el socialismo y el marxismo a secas, véase Gargarella (2002).

234 Andamios 
como el exceso dislocatorio que debe ser ocultado en una sociedad de mercado. Una vez que "atravesamos" el núcleo de esta "fantasía ideológica" $(\bullet \bullet \bullet k, 2003)$ se revela la opacidad que da forma a la cooperación productiva, una situación en la cual todos somos instrumentos del capital que es, por definición, el más ocioso entre los ociosos.

\section{CONCLUSIÓN}

En este trabajo he presentado una serie de afirmaciones que pueden ser resumidas en una sola tesis: la improductividad y el ocio constituyen dos momentos de extimidad (o de exterioridad-intima) de la estructura de la cooperación social productiva. ${ }^{10}$ Sostuve que tanto para Marx como para Rawls — sin olvidar a Schmidtz- la hipótesis ontológica que fundamenta la estructura de los arreglos productivos (para que ésta sea posible, los improductivos deben quedar fuera de sus límites) justifica la hipótesis normativa según la cual los improductivos no son agentes dignos de la justicia. Una justificación análoga se encuentra en el control razonable del ocio, pues su presencia excesiva más allá de la férrea organización de la cooperación social capitalista exige su exclusión. La otra cara de esta hipótesis es la desaprobación normativa de un tiempo libre extendido más allá de lo permitido por los tiempos rígidos que determina la lógica del mercado. En las sociedades globalizadas contemporáneas, caracterizadas por una multitud de improductivos sin recursos y de pocos aventajados que pueden aumentar con mayor libertad un tiempo libre pleno de comodidades, resulta difícil seguir entendiendo el ocio como algo excepcional dentro de la estructura de la cooperación social. Antes bien, en este trabajo me he inclinado por presentarlo como el exceso

\footnotetext{
${ }^{10}$ La noción de "extimidad" es utilizada por Lacan para dar cuenta, entre otras cuestiones, del estatuto de lo Real (lo que aquí hemos llamado "lo heterogéneo"): por un lado, lo Real es exterior a todo sistema de simbolización pero, por otro lado, esa dislocación sólo puede mostrarse internamente a través de las fallas de lo Simbólico. Sobre esto, véase Laclau (2003: 70-79).
} 
fantasmático que "siempre está allî" a pesar de que permanece (o debe permanecer) lo más oculto posible. ${ }^{11}$

BiBLIOgRAFíA

BARRY, B. (2001), Teorías de la justicia. Barcelona: Gedisa.

Bataille, G. (2000), "The Psichological Structure of Fascism" en Fred Botting y Scott Wilson (comps.), The Bataille Reader. Oxford: Blackwell, pp. 122-146.

Cohen, G. (2001), Si eres igualitarista, ¿cómo es que eres tan rico? Barcelona: Paidós.

COPJec, J. (2006), Imaginemos que la mujer no existe. Ética y sublimación. Buenos Aires: Fondo de Cultura Económica.

DerRidA, Jacques (2003a), Espectros de Marx. El estado de la deuda, el trabajo del duelo y la nueva internacional. Madrid: Trotta. (2003b), Márgenes de la filosofía. Madrid: Cátedra.

Dworkin, R. (2003), Virtud soberana. La teoría y la práctica de la igualdad. Barcelona: Paidós.

Gargarella, R. (2002), "Liberalismo frente a socialismo" en Atilio Borón y Álvaro de Vita (comps.), Teoría y filosofía política. La recuperación de los clásicos en el debate latinoamericano. Buenos Aires: CLACSO.

Laclau, E. (1988), "Politics and the Limits of Modernity" en Andrew Ross (comp.), Universal Abandon? The Politics of Postmodernism. Minnesota: University of Minnesota Press. (1993), Nuevas reflexiones sobre la revolución de nuestro tiempo. Buenos Aires: Nueva Visión. (2002), Misticismo, retórica y política. Buenos Aires: Fondo de Cultura Económica.

${ }^{11}$ Este trabajo se ha visto beneficiado por los comentarios, sugerencias y críticas de Julio Aibar, Dante Avaro, Carlos Durán, Rebeca Gaytan y Ricardo Nava. A todos ellos va mi agradecimiento, aunque evidentemente no cabe responsabilizarlos por los argumentos que aquí se presentan. 
(2003), "Identidad y hegemonía: el rol de la universalidad en la constitución de lógicas políticas" en Judith Butler, Ernesto Laclau y Slavoj •i•ek, Contingencia, hegemonía, universalidad. Diálogos contemporáneos en la izquierda. Buenos Aires: Fondo de Cultura Económica.

(2005), La razón populista. Buenos Aires: Fondo de Cultura Económica.

Lafargue, P. (1991), El derecho a la pereza. Madrid: Fundamentos.

MacIntyre, A. (2001), Tras la virtud. Barcelona: Crítica.

Macpherson, C. B. (1991), Ascenso y caída de la justicia económica y otros ensayos. Buenos Aires: Manantial.

MarX, C. (1978), "El dieciocho Brumario de Luis Bonaparte" en Carlos Marx y Federico Engels, Obras escogidas. Moscú: Progreso.

PARIJs, Ph. van (1993), ¿Qué es una sociedad justa? Introducción a la práctica de la filosofía politica. Barcelona: Ariel.

RAWLS, J. (1996a), "La justicia como equidad: política, no metafísica" en Agora, año 2, núm. 4, verano. Buenos Aires, pp. 27-50. (1996b), Liberalismo político. México: Fondo de Cultura Económica.

(1997), Teoría de la justicia. México: Fondo de Cultura Económica. (1999), Justicia como equidad. Materiales para una teoría de la justicia. Madrid: Tecnos.

(2002), La justicia como equidad. Una reformulación. Barcelona: Paidós.

Schmidtz, D. (2000), "Asumir la responsabilidad" en David Schmidtz y Robert Goodin, Bienestar social y responsabilidad individual. Madrid: Cambridge University Press.

Walzer, M. (1997), Las esferas de la justicia. Una defensa del pluralismo y la igualdad. México: Fondo de Cultura Económica.

- ${ }^{\bullet E K}$, Slavoj (2003), El sublime objeto de la ideología. Buenos Aires: Siglo XXI. 